Acta Crystallographica Section E

Structure Reports

Online

ISSN 1600-5368

\section{A second monoclinic polymorph of 2,4-dimethylanilinium chloride}

\section{Soudeh Hossein Zadeh, ${ }^{\mathrm{a}}$ Mohamad Reza Talei Bavil Olyai, $^{b *}$ Hossein Biglari Mazlaghani ${ }^{\mathbf{a}}$ and Behrouz Notash $^{\mathrm{c}}$}

a Department of Chemistry, Islamic Azad University, Karaj Branch, Karaj, Iran, ${ }^{\mathbf{b}}$ Department of Chemistry, Islamic Azad University, South Tehran Branch, Tehran, Iran, and ${ }^{\mathbf{c}}$ Department of Chemistry, Shahid Beheshti University, G. C., Evin, Tehran 1983963113, Iran

Correspondence e-mail: talei3@gmail.com

Received 14 October 2011; accepted 29 October 2011

Key indicators: single-crystal X-ray study; $T=298 \mathrm{~K}$; mean $\sigma(\mathrm{C}-\mathrm{C})=0.002 \AA$; $R$ factor $=0.042 ; w R$ factor $=0.104 ;$ data-to-parameter ratio $=21.7$.

A second monoclinic polymorph of 2,4-dimethylanilinium chloride, $\mathrm{C}_{8} \mathrm{H}_{12} \mathrm{~N}^{+} \cdot \mathrm{Cl}^{-}$, (I), is reported. The unit-cell dimensions differ from those of the first reported monoclinic form, (II) [Yao (2010). Acta Cryst. E66, o1563]. Nevertheless, both compounds crystallize in the monoclinic space group $P 2_{1} / n$. As in (II), the protonated amine group in (I) acts as a hydrogenbond donor to the chloride ion, forming three $\mathrm{N}-\mathrm{H} \cdots \mathrm{Cl}$ hydrogen bonds. The result is a two-dimensional network in the $a c$ plane. The difference in the hydrogen-bond pattern is that in (I) only 12-membered rings are formed whereas in (II), eight-membered and 16-membered rings are formed.

\section{Related literature}

For another monoclinic polymorph of the title compound, see: Yao (2010). For properties of compounds containing inorganic anions and organic cations, see: Masse et al. (1993); Xiao et al. (2005).

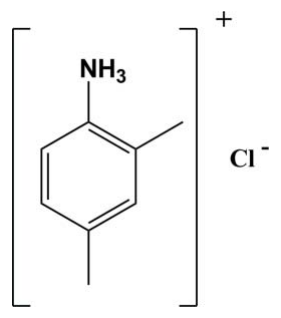

\section{Experimental}

Crystal data

$\mathrm{C}_{8} \mathrm{H}_{12} \mathrm{~N}^{+} \cdot \mathrm{Cl}^{-}$

$M_{r}=157.64$

Monoclinic, $P 2_{1} / n$

$a=5.3651(11) \AA$

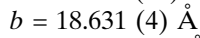

$c=8.5428$ (17)

$\beta=94.48(3)^{\circ}$

$$
\begin{aligned}
& V=851.3(3) \AA^{3} \\
& Z=4 \\
& \text { Mo } K \alpha \text { radiation } \\
& \mu=0.38 \mathrm{~mm}^{-1} \\
& T=298 \mathrm{~K} \\
& 0.45 \times 0.4 \times 0.34 \mathrm{~mm}
\end{aligned}
$$

\section{Data collection}

Stoe IPDS 2T diffractometer

5557 measured reflections

2276 independent reflections

1838 reflections with $I>2 \sigma(I)$

$R_{\text {int }}=0.031$

\section{Refinement}

$R\left[F^{2}>2 \sigma\left(F^{2}\right)\right]=0.042$

$w R\left(F^{2}\right)=0.104$

$S=1.09$

2276 reflections

105 parameters

$$
\begin{aligned}
& \mathrm{H} \text { atoms treated by a mixture of } \\
& \text { independent and constrained } \\
& \text { refinement } \\
& \Delta \rho_{\max }=0.22 \mathrm{e}^{-3} \\
& \Delta \rho_{\min }=-0.17 \mathrm{e}^{-3}
\end{aligned}
$$

Table 1

Hydrogen-bond geometry $\left(\AA{ }^{\circ}\right)$.

\begin{tabular}{lllll}
\hline$D-\mathrm{H} \cdots A$ & $D-\mathrm{H}$ & $\mathrm{H} \cdots A$ & $D \cdots A$ & $D-\mathrm{H} \cdots A$ \\
\hline $\mathrm{N} 1-\mathrm{H} 1 A \cdots \mathrm{Cl} 1^{\mathrm{i}}$ & $0.91(2)$ & $2.28(2)$ & $3.1721(17)$ & $164.4(17)$ \\
$\mathrm{N} 1-\mathrm{H} 1 B \cdots \mathrm{Cl} 1^{\text {ii }}$ & $0.88(2)$ & $2.35(2)$ & $3.2228(17)$ & $172.1(18)$ \\
$\mathrm{N} 1-\mathrm{H} 1 C \cdots \mathrm{Cl} 1^{\text {iii }}$ & $0.94(2)$ & $2.23(2)$ & $3.1630(16)$ & $172.8(19)$ \\
\hline
\end{tabular}

Symmetry codes: (i) $x+\frac{1}{2},-y+\frac{3}{2}, z+\frac{1}{2}$; (ii) $x+\frac{3}{2},-y+\frac{3}{2}, z+\frac{1}{2}$; (iii) $x+1, y, z$.

Data collection: $X$-AREA (Stoe \& Cie, 2005); cell refinement: $X$ $A R E A$; data reduction: $X$ - $A R E A$; $\operatorname{program}(\mathrm{s})$ used to solve structure: SHELXS97 (Sheldrick, 2008); program(s) used to refine structure: SHELXL97 (Sheldrick, 2008); molecular graphics: ORTEP-3 for Windows (Farrugia, 1997); software used to prepare material for publication: Win $G X$ (Farrugia, 1999).

The authors wish to acknowledge Islamic Azad University, Karaj Branch for financial support.

Supplementary data and figures for this paper are available from the IUCr electronic archives (Reference: BT5679).

\title{
References
}

Farrugia, L. J. (1997). J. Appl. Cryst. 30, 565. Farrugia, L. J. (1999). J. Appl. Cryst. 32, 837-838.

Masse, R., Bagieu-Beucher, M., Pecault, J., Levy, J. P. \& Zyss, J. (1993). Nonlin. Opt. 5, 413-423.

Sheldrick, G. M. (2008). Acta Cryst. A64, 112-122.

Stoe \& Cie (2005). $X$-AREA and $X$-RED32. Stoe \& Cie, Darmstadt, Germany. Xiao, D., An, H., Wang, E. \& Xu, L. (2005). J. Mol. Struct. 738, 217-225.

Yao, J.-Y. (2010). Acta Cryst. E66, o1563. 


\section{supporting information}

Acta Cryst. (2011). E67, o3190 [https://doi.org/10.1107/S160053681104551X]

\section{A second monoclinic polymorph of 2,4-dimethylanilinium chloride}

\section{Soudeh Hossein Zadeh, Mohamad Reza Talei Bavil Olyai, Hossein Biglari Mazlaghani and Behrouz Notash}

\section{S1. Comment}

The combination of organic cations and inorganic anions is important in material science and chemistry because of their abilities to join the properties of organic and inorganic molecules, exhibiting some interesting crystal structures with special properties, such as luminescence, magnetism, and multifunctional properties (Masse et al., 1993). Furthermore these hybrid materials have a great interest due to their numerous varieties of intriguing structural topologies (Xiao et al., 2005).

The title compound, (I), is a second monoclinic polymorph of the structure of 2,4-dimethylanilinium chloride which crystallizes in the space group $P 2_{1} / n$. A different structure, (II), in the space group $P 2_{1} / c$ was described earlier by (Yao, 2010).

The asymmetric unit of the title compound contains one 2,4-dimethylanilinium cation and one chloride anion (Fig. 1). The cell parameters of the current monoclinic polymorph vary significantly from the earlier polymorph $[a=9.4739(19)$ $\AA, b=9.894$ (2) $\AA, c=9.6709$ (19) $\AA$ and $\left.\beta=96.31(3)^{\circ}\right]$. The $\mathrm{C}-\mathrm{N}$ bond length is 1.465 (2) $\AA$. The $\mathrm{C}-\mathrm{N}$ bond length for previous crystal structure was given 1.466 (3) $\AA$ (Yao, 2010). Inside lattice, each $\mathrm{Cl}^{-}$anion is linked to three organic species through $\mathrm{N}-\mathrm{H} \cdots \mathrm{Cl}$ hydrogen bonds (Table 1).

The two-dimensional arrangement of the chloride anions and 2,4-dimethylanilinum cations in the unit cell is shown in Fig. 2 .

\section{S2. Experimental}

An ethanolic solution of 2,4-dimethylaniline $(1.21 \mathrm{~g}, 10 \mathrm{mmol}$ in $10 \mathrm{ml})$ was added dropwise to a magnetically stirred of aqueous hydrochloric acid solution $(1.01 \mathrm{~g}, 10 \mathrm{mmol})$ a 1:1 molar ratio. The achieved solution is then filtered to eliminate the colorless crystals precipitated formed and then stirred for $2 \mathrm{hrs}$. After stirring, the obtained solution was slowly evaporated at room temperature over several days resulting in the formation of 2,4-dimethylanilinium chloride crystals.

\section{S3. Refinement}

The hydrogen atoms of the protonated nitrogen were found in difference Fourier map and refined isotropically. The $\mathrm{C}-\mathrm{H}$ protons were positioned geometrically and refined as riding atoms with $\mathrm{C}-\mathrm{H}=0.93 \AA$ and $U$ iso( $\mathrm{H})=1.2 U$ eq $(\mathrm{C})$ for aromatic $\mathrm{C}-\mathrm{H}$ and $\mathrm{C}-\mathrm{H}=0.96 \AA$ and $\operatorname{Uiso}(\mathrm{H})=1.5 \operatorname{Ueq}(\mathrm{C})$ for methyl groups. 


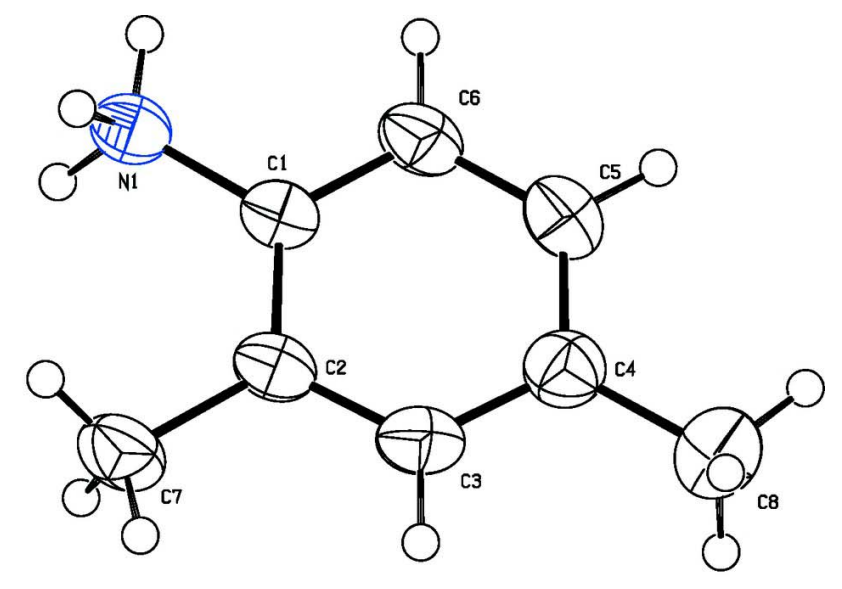

CL 1

Figure 1

The molecular structure of the title compound with the atom-numbering scheme. Displacement ellipsoids are drawn at the $50 \%$ probability level.

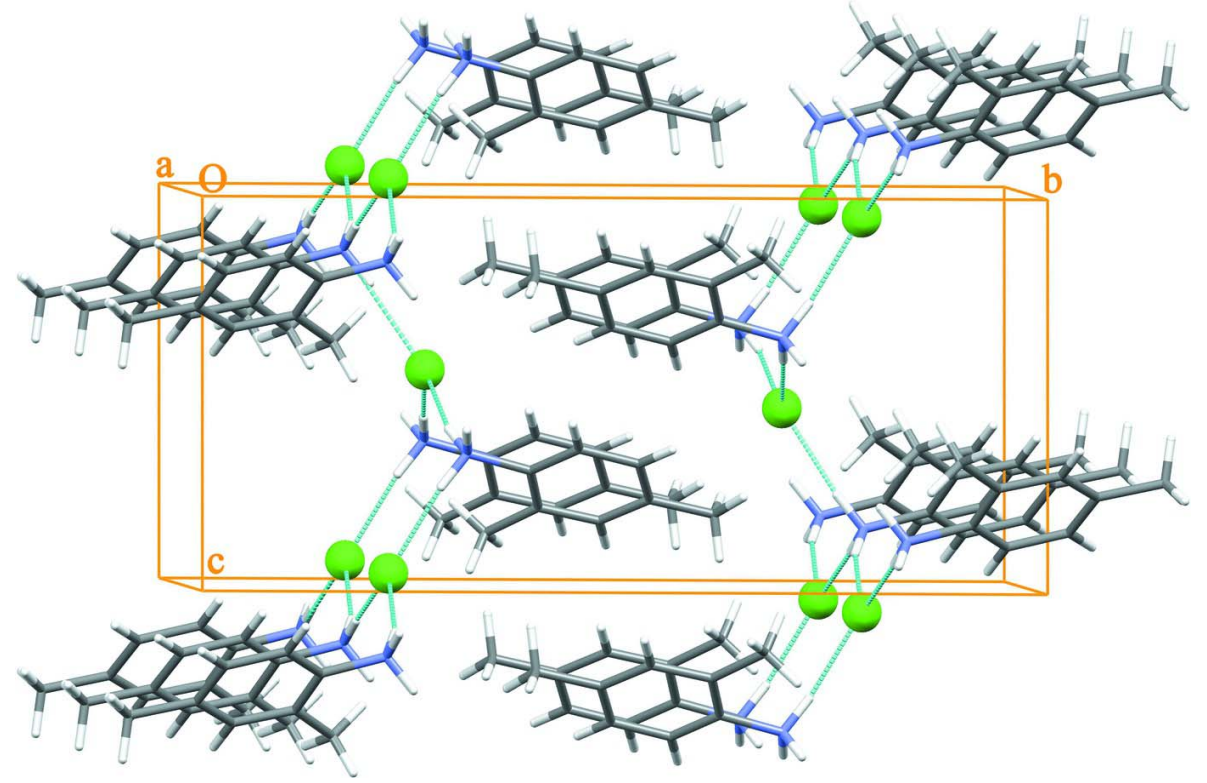

Figure 2

Packing diagram of the title compound showing intermolecular $\mathrm{N}-\mathrm{H} \cdots \mathrm{Cl}$ hydrogen bonding.

2,4-dimethylanilinium chloride

Crystal data

$\mathrm{C}_{8} \mathrm{H}_{12} \mathrm{~N}^{+} \cdot \mathrm{Cl}^{-}$

$M_{r}=157.64$

Monoclinic, $P 2_{1} / n$

Hall symbol: -P $2 \mathrm{yn}$

$a=5.3651$ (11) $\AA$

$b=18.631(4) \AA$

$c=8.5428(17) \AA$

$\beta=94.48(3)^{\circ}$

$V=851.3(3) \AA^{3}$

$Z=4$

$F(000)=336$

$D_{\mathrm{x}}=1.230 \mathrm{Mg} \mathrm{m}^{-3}$

Mo $K \alpha$ radiation, $\lambda=0.71073 \AA$

Cell parameters from 2276 reflections 
$\theta=2.2-29.1^{\circ}$

$\mu=0.38 \mathrm{~mm}^{-1}$

$T=298 \mathrm{~K}$

\section{Data collection}

Stoe IPDS 2T

diffractometer

Radiation source: fine-focus sealed tube

Graphite monochromator

Detector resolution: $0.15 \mathrm{~mm}$ pixels $\mathrm{mm}^{-1}$

rotation method scans

5557 measured reflections

\section{Refinement}

Refinement on $F^{2}$

Least-squares matrix: full

$R\left[F^{2}>2 \sigma\left(F^{2}\right)\right]=0.042$

$w R\left(F^{2}\right)=0.104$

$S=1.09$

2276 reflections

105 parameters

0 restraints

Primary atom site location: structure-invariant direct methods
Block, colorless

$0.45 \times 0.4 \times 0.34 \mathrm{~mm}$

2276 independent reflections

1838 reflections with $I>2 \sigma(I)$

$R_{\text {int }}=0.031$

$\theta_{\text {max }}=29.1^{\circ}, \theta_{\min }=2.2^{\circ}$

$h=-7 \rightarrow 6$

$k=-22 \rightarrow 25$

$l=-11 \rightarrow 11$

Secondary atom site location: difference Fourier map

Hydrogen site location: inferred from neighbouring sites

$\mathrm{H}$ atoms treated by a mixture of independent and constrained refinement

$w=1 /\left[\sigma^{2}\left(F_{\mathrm{o}}^{2}\right)+(0.0462 P)^{2}+0.1562 P\right]$ where $P=\left(F_{\mathrm{o}}^{2}+2 F_{\mathrm{c}}^{2}\right) / 3$

$(\Delta / \sigma)_{\max }<0.001$

$\Delta \rho_{\max }=0.22$ e $\AA^{-3}$

$\Delta \rho_{\min }=-0.17$ e $\AA^{-3}$

\section{Special details}

Geometry. All e.s.d.'s (except the e.s.d. in the dihedral angle between two 1.s. planes) are estimated using the full covariance matrix. The cell e.s.d.'s are taken into account individually in the estimation of e.s.d.'s in distances, angles and torsion angles; correlations between e.s.d.'s in cell parameters are only used when they are defined by crystal symmetry. An approximate (isotropic) treatment of cell e.s.d.'s is used for estimating e.s.d.'s involving 1.s. planes.

Refinement. Refinement of $F^{2}$ against ALL reflections. The weighted $R$-factor $w R$ and goodness of fit $S$ are based on $F^{2}$, conventional $R$-factors $R$ are based on $F$, with $F$ set to zero for negative $F^{2}$. The threshold expression of $F^{2}>\sigma\left(F^{2}\right)$ is used only for calculating $R$-factors $(\mathrm{gt})$ etc. and is not relevant to the choice of reflections for refinement. $R$-factors based on $F^{2}$ are statistically about twice as large as those based on $F$, and $R$-factors based on ALL data will be even larger.

Fractional atomic coordinates and isotropic or equivalent isotropic displacement parameters $\left(\AA^{2}\right)$

\begin{tabular}{lllll}
\hline & $x$ & $y$ & $z$ & $U_{\text {iso }} * / U_{\text {eq }}$ \\
\hline C8 & $0.9245(5)$ & $0.39088(11)$ & $0.1976(3)$ & $0.0692(6)$ \\
H8A & 1.0616 & 0.3635 & 0.2452 & $0.104^{*}$ \\
H8B & 0.9148 & 0.3842 & 0.0858 & $0.104^{*}$ \\
H8C & 0.7715 & 0.3750 & 0.2376 & $0.104^{*}$ \\
C7 & $0.6327(3)$ & $0.64679(10)$ & $0.1382(2)$ & $0.0533(4)$ \\
H7A & 0.6040 & 0.6834 & 0.2139 & $0.080^{*}$ \\
H7B & 0.4783 & 0.6227 & 0.1078 & $0.080^{*}$ \\
H7C & 0.6969 & 0.6683 & 0.0475 & $0.080^{*}$ \\
C11 & $0.08615(7)$ & $0.78443(2)$ & $0.04928(5)$ & $0.04796(14)$ \\
N1 & $1.0561(3)$ & $0.68899(8)$ & $0.35343(16)$ & $0.0406(3)$ \\
C1 & $1.0245(3)$ & $0.61337(8)$ & $0.30934(16)$ & $0.0367(3)$ \\
C2 & $0.8198(3)$ & $0.59328(9)$ & $0.20976(17)$ & $0.0407(3)$ \\
C3 & $0.7952(3)$ & $0.52034(10)$ & $0.17611(19)$ & $0.0477(4)$ \\
H3 & 0.6585 & 0.5053 & 0.1107 & $0.057^{*}$
\end{tabular}




\begin{tabular}{lllll} 
C5 & $1.1683(3)$ & $0.49210(10)$ & $0.3321(2)$ & $0.0502(4)$ \\
H5 & 1.2861 & 0.4589 & 0.3719 & $0.060^{*}$ \\
C4 & $0.9645(3)$ & $0.46926(9)$ & $0.23530(19)$ & $0.0478(4)$ \\
C6 & $1.1980(3)$ & $0.56416(9)$ & $0.37005(19)$ & $0.0458(4)$ \\
H6 & 1.3341 & 0.5792 & 0.4360 & $0.055^{*}$ \\
H1A & $0.932(4)$ & $0.7056(11)$ & $0.411(3)$ & $0.057(6)^{*}$ \\
H1C & $1.060(4)$ & $0.7208(11)$ & $0.268(3)$ & $0.062(6)^{*}$ \\
H1B & $1.192(5)$ & $0.6971(11)$ & $0.415(2)$ & $0.057(6)^{*}$ \\
\hline
\end{tabular}

Atomic displacement parameters $\left(\AA^{2}\right)$

\begin{tabular}{lllllll}
\hline & $U^{11}$ & $U^{22}$ & $U^{33}$ & $U^{12}$ & $U^{13}$ & $U^{23}$ \\
\hline C8 & $0.0840(16)$ & $0.0542(12)$ & $0.0681(12)$ & $0.0007(11)$ & $-0.0025(11)$ & $-0.0061(9)$ \\
C7 & $0.0395(9)$ & $0.0614(11)$ & $0.0569(10)$ & $0.0008(8)$ & $-0.0108(7)$ & $0.0054(8)$ \\
C11 & $0.0356(2)$ & $0.0578(3)$ & $0.0501(2)$ & $-0.00034(18)$ & $0.00128(14)$ & $0.01375(18)$ \\
N1 & $0.0323(7)$ & $0.0494(8)$ & $0.0397(7)$ & $-0.0023(6)$ & $0.0003(5)$ & $0.0018(6)$ \\
C1 & $0.0299(7)$ & $0.0462(8)$ & $0.0342(6)$ & $-0.0031(6)$ & $0.0036(5)$ & $0.0034(6)$ \\
C2 & $0.0325(7)$ & $0.0526(9)$ & $0.0366(7)$ & $-0.0016(7)$ & $0.0001(5)$ & $0.0035(6)$ \\
C3 & $0.0437(9)$ & $0.0567(10)$ & $0.0415(8)$ & $-0.0067(7)$ & $-0.0051(6)$ & $-0.0019(7)$ \\
C5 & $0.0442(9)$ & $0.0532(10)$ & $0.0525(9)$ & $0.0065(8)$ & $-0.0007(7)$ & $0.0080(7)$ \\
C4 & $0.0530(10)$ & $0.0499(9)$ & $0.0406(8)$ & $-0.0012(8)$ & $0.0048(7)$ & $0.0010(7)$ \\
C6 & $0.0345(8)$ & $0.0553(10)$ & $0.0462(8)$ & $-0.0017(7)$ & $-0.0056(6)$ & $0.0062(7)$ \\
& & & & & &
\end{tabular}

Geometric parameters $\left(\AA,{ }^{\circ}\right)$

\begin{tabular}{llll}
\hline $\mathrm{C} 8-\mathrm{C} 4$ & $1.507(3)$ & $\mathrm{N} 1-\mathrm{H} 1 \mathrm{~B}$ & $0.88(2)$ \\
$\mathrm{C} 8-\mathrm{H} 8 \mathrm{~A}$ & 0.9600 & $\mathrm{C} 1-\mathrm{C} 6$ & $1.379(2)$ \\
$\mathrm{C} 8-\mathrm{H} 8 \mathrm{~B}$ & 0.9600 & $\mathrm{C} 1-\mathrm{C} 2$ & $1.387(2)$ \\
$\mathrm{C} 8-\mathrm{H} 8 \mathrm{C}$ & 0.9600 & $\mathrm{C} 2-\mathrm{C} 3$ & $1.393(2)$ \\
$\mathrm{C} 7-\mathrm{C} 2$ & $1.509(2)$ & $\mathrm{C} 3-\mathrm{C} 4$ & $1.383(2)$ \\
$\mathrm{C} 7-\mathrm{H} 7 \mathrm{~A}$ & 0.9600 & $\mathrm{C} 3-\mathrm{H} 3$ & 0.9300 \\
$\mathrm{C} 7-\mathrm{H} 7 \mathrm{~B}$ & 0.9600 & $\mathrm{C} 5-\mathrm{C} 4$ & $1.385(3)$ \\
$\mathrm{C} 7-\mathrm{H} 7 \mathrm{C}$ & 0.9600 & $\mathrm{C} 5-\mathrm{C} 6$ & $1.387(3)$ \\
$\mathrm{N} 1-\mathrm{C} 1$ & $1.465(2)$ & $\mathrm{C} 5-\mathrm{H} 5$ & 0.9300 \\
$\mathrm{~N} 1-\mathrm{H} 1 \mathrm{~A}$ & $0.91(2)$ & $\mathrm{C} 6-\mathrm{H} 6$ & 0.9300 \\
$\mathrm{~N} 1-\mathrm{H} 1 \mathrm{C}$ & $0.94(2)$ & & \\
& & & $122.02(15)$ \\
$\mathrm{C} 4-\mathrm{C} 8-\mathrm{H} 8 \mathrm{~A}$ & 109.5 & $\mathrm{C} 6-\mathrm{C} 1-\mathrm{C} 2$ & $118.79(14)$ \\
$\mathrm{C} 4-\mathrm{C} 8-\mathrm{H} 8 \mathrm{~B}$ & 109.5 & $\mathrm{C} 6-\mathrm{C} 1-\mathrm{N} 1$ & $119.18(14)$ \\
$\mathrm{H} 8 \mathrm{~A}-\mathrm{C} 8-\mathrm{H} 8 \mathrm{~B}$ & 109.5 & $\mathrm{C} 2-\mathrm{C} 1-\mathrm{N} 1$ & $116.57(15)$ \\
$\mathrm{C} 4-\mathrm{C} 8-\mathrm{H} 8 \mathrm{C}$ & 109.5 & $\mathrm{C} 1-\mathrm{C} 2-\mathrm{C} 3$ & $122.66(15)$ \\
$\mathrm{H} 8 \mathrm{~A}-\mathrm{C} 8-\mathrm{H} 8 \mathrm{C}$ & 109.5 & $\mathrm{C} 1-\mathrm{C} 2-\mathrm{C} 7$ & $120.76(15)$ \\
$\mathrm{H} 8 \mathrm{~B}-\mathrm{C} 8-\mathrm{H} 8 \mathrm{C}$ & 109.5 & $\mathrm{C} 4-\mathrm{C} 2-\mathrm{C} 3-\mathrm{C} 2$ & $123.15(16)$ \\
$\mathrm{C} 2-\mathrm{C} 7-\mathrm{H} 7 \mathrm{~A}$ & 109.5 & $\mathrm{C} 4-\mathrm{C} 3-\mathrm{H} 3$ & 118.4 \\
$\mathrm{C} 2-\mathrm{C} 7-\mathrm{H} 7 \mathrm{~B}$ & 109.5 & $\mathrm{C} 2-\mathrm{C} 3-\mathrm{H} 3$ & 118.4 \\
$\mathrm{H} 7 \mathrm{~A}-\mathrm{C} 7-\mathrm{H} 7 \mathrm{~B}$ & 109.5 & $\mathrm{C} 4-\mathrm{C} 5-\mathrm{C} 56$ & $120.51(16)$ \\
$\mathrm{C} 2-\mathrm{C} 7-\mathrm{H} 7 \mathrm{C}$ & 109.5 & & 119.7 \\
$\mathrm{H} 7 \mathrm{~A}-\mathrm{C} 7-\mathrm{H} 7 \mathrm{C}$ & 109.5 & &
\end{tabular}




$\begin{array}{llll}\mathrm{H} 7 \mathrm{~B}-\mathrm{C} 7-\mathrm{H} 7 \mathrm{C} & 109.5 & \mathrm{C} 6-\mathrm{C} 5-\mathrm{H} 5 & 119.7 \\ \mathrm{C} 1-\mathrm{N} 1-\mathrm{H} 1 \mathrm{~A} & 112.9(13) & \mathrm{C} 3-\mathrm{C} 4-\mathrm{C} 5 & 118.14(16) \\ \mathrm{C} 1-\mathrm{N} 1-\mathrm{H} 1 \mathrm{C} & 114.4(12) & \mathrm{C} 3-\mathrm{C} 4-\mathrm{C} 8 & 120.66(17) \\ \mathrm{H} 1 \mathrm{~A}-\mathrm{N} 1-\mathrm{H} 1 \mathrm{C} & 105.4(18) & \mathrm{C} 5-\mathrm{C} 4-\mathrm{C} 8 & 121.19(18) \\ \mathrm{C} 1-\mathrm{N} 1-\mathrm{H} 1 \mathrm{~B} & 113.2(13) & \mathrm{C} 1-\mathrm{C} 6-\mathrm{C} 5 & 119.59(15) \\ \mathrm{H} 1 \mathrm{~A}-\mathrm{N} 1-\mathrm{H} 1 \mathrm{~B} & 103.1(18) & \mathrm{C} 1-\mathrm{C} 6-\mathrm{H} 6 & 120.2 \\ \mathrm{H} 1 \mathrm{C}-\mathrm{N} 1-\mathrm{H} 1 \mathrm{~B} & 106.9(18) & \mathrm{C} 5-\mathrm{C} 6-\mathrm{H} 6 & 178.59(17) \\ \mathrm{C} 6-\mathrm{C} 1-\mathrm{C} 2-\mathrm{C} 3 & & \mathrm{C} 2-\mathrm{C} 3-\mathrm{C} 4-\mathrm{C} 8 & 1.1(2) \\ \mathrm{N} 1-\mathrm{C} 1-\mathrm{C} 2-\mathrm{C} 3 & \mathrm{C} 6-\mathrm{C} 5-\mathrm{C} 4-\mathrm{C} 3 & -177.79(17) \\ \mathrm{C} 6-\mathrm{C} 1-\mathrm{C} 2-\mathrm{C} 7 & \mathrm{C} 6-\mathrm{C} 5-\mathrm{C} 4-\mathrm{C} 8 & 178.72(15) \\ \mathrm{N} 1-\mathrm{C} 1-\mathrm{C} 2-\mathrm{C} 7 & \mathrm{C} 2-\mathrm{C} 1-\mathrm{C} 6-\mathrm{C} 5 & -0.7(3) \\ \mathrm{C} 1-\mathrm{C} 2-\mathrm{C} 3-\mathrm{C} 4 & -177.97(14) & \mathrm{N} 1-\mathrm{C} 1-\mathrm{C} 6-\mathrm{C} 5 & \mathrm{C} 4-\mathrm{C} 5-\mathrm{C} 6-\mathrm{C} 1 \\ \mathrm{C} 7-\mathrm{C} 2-\mathrm{C} 3-\mathrm{C} 4 & 2.9(2) & & \end{array}$

Hydrogen-bond geometry $\left(\AA,{ }^{\circ}\right)$

\begin{tabular}{lllll}
\hline$D-\mathrm{H} \cdots A$ & $D-\mathrm{H}$ & $\mathrm{H} \cdots A$ & $D \cdots A$ & $D-\mathrm{H} \cdots A$ \\
\hline $\mathrm{N} 1-\mathrm{H} 1 A \cdots \mathrm{C} 11^{\mathrm{i}}$ & $0.91(2)$ & $2.28(2)$ & $3.1721(17)$ & $164.4(17)$ \\
$\mathrm{N} 1-\mathrm{H} 1 B \cdots \mathrm{C} 11^{\mathrm{ii}}$ & $0.88(2)$ & $2.35(2)$ & $3.2228(17)$ & $172.1(18)$ \\
$\mathrm{N} 1-\mathrm{H} 1 C \cdots \mathrm{C} 11^{\mathrm{ii}}$ & $0.94(2)$ & $2.23(2)$ & $3.1630(16)$ & $172.8(19)$ \\
\hline
\end{tabular}

Symmetry codes: (i) $x+1 / 2,-y+3 / 2, z+1 / 2$; (ii) $x+3 / 2,-y+3 / 2, z+1 / 2$; (iii) $x+1, y, z$. 